\title{
Optical and Terahertz Characterization of Be-Doped GaAs/AlAs Multiple Quantum Wells
}

\author{
B. Čenchavičius ${ }^{a}$, J. Kavaliauskas ${ }^{a, *}$, G. Krivaité $\dot{K}^{a}$, \\ D. Seliuta ${ }^{a}$, E. Šrrmulis ${ }^{a}$, J. Devenson ${ }^{a}$, G. VAlušis $^{a}$, \\ B. Sherliker ${ }^{b}$, M.P. HAlsall ${ }^{b}$, M.J. Steer ${ }^{c}$ \\ AND P. HARRISON ${ }^{d}$ \\ ${ }^{a}$ Semiconductor Physics Institute, A. Goštauto 11, 01108, Vilnius, Lithuania \\ ${ }^{b}$ Department of Physics, UMIST, Manchester M60 1QD, United Kingdom \\ ${ }^{c}$ Department of Electronic and Electrical Engineering, University of Sheffield \\ Sheffield S1 3JD, United Kingdom \\ ${ }^{d}$ IMP, School of Electronic and Electrical Engineering, University of Leeds \\ Leeds LS2 9JT, United Kingdom
}

We report on optical, photoreflectance and surface photovoltage, as well as terahertz photocurrent investigation of Be-doped GaAs/AlAs multiple quantum wells at room and liquid helium temperatures, respectively. From the Franz-Keldysh oscillations observed in photoreflectance spectra we determine built-in electric fields within the structure. Interband transition energies calculated by the transfer matrix method are in qualitative agreement with experimentally determined values for the samples having various, from $2 \times 10^{10}$ up to $2.5 \times 10^{12} \mathrm{~cm}^{-2}$, Be doping densities. The photocurrent observed in the range of $5.4-7.3 \mathrm{THz}$ we associate with photoionization of Be-acceptor states.

PACS numbers: 78.66.--w, 85.30.De

\section{Introduction}

Rapid evolution of $\mathrm{THz}$ electronics has stimulated a search for new solutions to realize terahertz devices. The engineering of shallow impurities levels by varying the width of a confining quantum well (QW) provides the possibility to use such

*corresponding author; e-mail: jk@pfi.lt 
structures for $\mathrm{THz}$ detectors/emitters [1]. The control of their energy level position and knowledge concerning the presence of internal electric fields and imperfections is therefore of particular importance in the design of such devices.

In this communication, we report both, optical and terahertz studies of Be $\delta$-doped GaAs/AlAs multiple QW structures. The GaAs/AlAs QW system is of special interest since it represents the maximum possible confinement for the acceptor states.

\section{Samples and experimental techniques}

The structures consist of GaAs QWs with different width $L_{\mathrm{w}}$ separated by AlAs barriers of thickness $5 \mathrm{~nm}$ grown by MBE on a semi-insulating GaAs substrate. The $\delta$-doped Be-layers were placed in the center of the wells. Parameters of the samples are given in the Table. The band diagram of $\delta$-doped multiple QW structures is shown in Fig. 1.

TABLE

Parameters of QWs: repeated period, QW width $-L_{\mathrm{w}}$, doping concentration $-P$.

\begin{tabular}{c|c|c|c}
\hline \hline Samples & Periods & $L_{\mathrm{w}}(\mathrm{nm})$ & $P\left(\mathrm{~cm}^{-2}\right)$ \\
\hline 1795 & 400 & 3 & $2 \times 10^{10}$ \\
1794 & 200 & 10 & $5 \times 10^{10}$ \\
1392 & 40 & 20 & $2.5 \times 10^{12}$ \\
1796 & a single $5 \mu \mathrm{m}$ thick epilayer (GaAs:Be) & $2 \times 10^{16}$
\end{tabular}

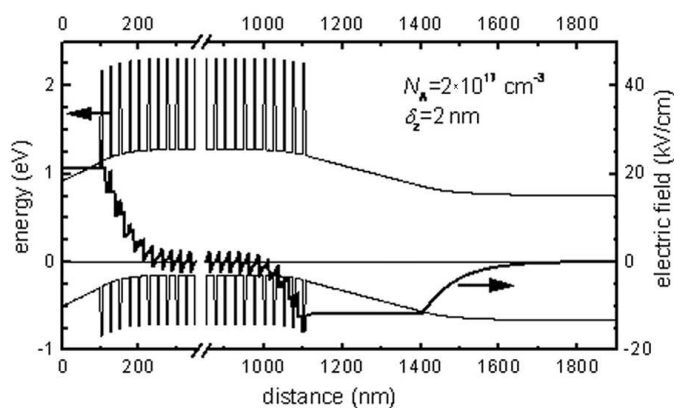

Fig. 1. Band diagram of Be $\delta$-doped multiple QW structures calculated by solving Schrödinger-Poisson equations.

We applied photoreflectance (PR) and surface photovoltage (SPV) spectroscopies as well as the terahertz photocurrent technique to characterize the structures. The PR measurements were performed using a $\mathrm{He}-\mathrm{Ne}$ (wavelength is $632.8 \mathrm{~nm}$ ) laser or light emitting diode (wavelength is $470 \mathrm{~nm}$ ) light beams as the 
modulation sources keeping pumping intensities below $2 \mathrm{~mW} \cdot \mathrm{cm}^{-2}$. In the terahertz photocurrent experiment we have used several lines of an optically pumped molecular $\mathrm{THz}$ laser in the spectral region of interest corresponding to the Be acceptor binding energy ( $28 \mathrm{meV}$ in bulk GaAs). The photocurrent measurements were performed in planar geometry in closed-cycle helium cryostat at $4 \mathrm{~K}$.

\section{Optical spectra}

Figure 2 shows the PR spectra in the range of $1.3-1.7 \mathrm{eV}$ for GaAs/AlAs QW samples with various well widths and doping concentrations. The PR spectrum from epitaxial $p$-GaAs layer is also shown for comparison. The characteristic

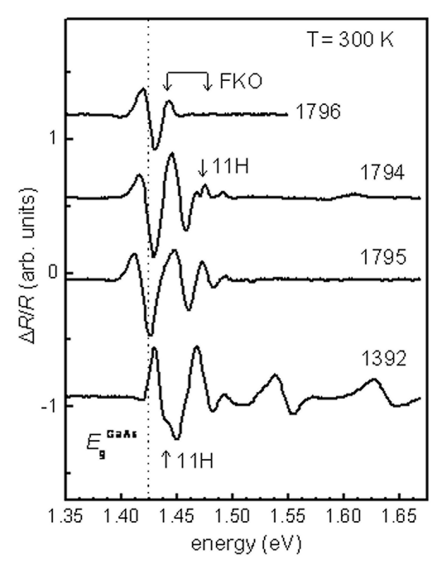

Fig. 2. PR spectra of GaAs/AlAs QW structures with different well widths at $300 \mathrm{~K}$. The main characteristic features are market by arrows. Data of Be-doped GaAs epilayer are given for comparison.

Franz-Keldysh oscillations (FKOs) are clearly seen above of the GaAs fundamental gap $E_{\mathrm{g}}(1.424 \mathrm{eV})$ for QW samples 1794, 1795, and the GaAs epilayer 1796, too. These spectral features indicate that internal electric field exists in the samples. The values of the built-in electric field in the GaAs buffer/cap layers estimated from the FKOs are within the range of $13-20 \mathrm{kV} / \mathrm{cm}$. For deeper understanding, we measured SPV spectra [2]. The results are given in Fig. 3. The SPV spectra, measured under the same conditions, exhibit features related to GaAs cap and/or buffer layers and QW structure itself. More specifically, the kink at about $1.42 \mathrm{eV}$ is related to the bulk GaAs band edge. At higher energies, characteristic discrete dips associated with excitonic transitions in QWs are clearly visible. The experimentally determined values of the interband transition energies were found to be in reasonable agreement with the calculated ones by the transfer matrix method. Also, it was observed that the broadening of spectral features depends on the doping level. Further, one must note that the spectra show the $n$-type behavior (positive sign) in spite of the presence of $p$-type band bending near the surface. 


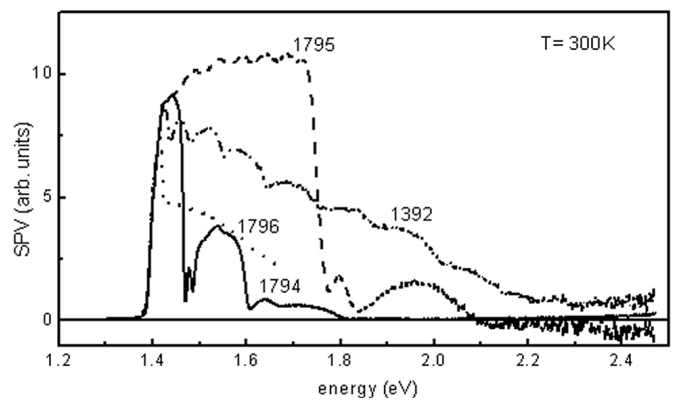

Fig. 3. Experimental SPV spectra of GaAs/AlAs multiple QW structures at $300 \mathrm{~K}$. Data of Be-doped GaAs epilayer are given for comparison.

These results can be explained in the following way: in the structures there are two oppositely directed electric fields, one is in the cap layer while the other one is located in the buffer layer (see Fig. 1). Therefore, the SPV signals from these structure regions should be of opposite signs [3]. As the total SPV is positive, it means that the SPV signal from the interface region of GaAs buffer layer is very strong, and that it conceals the signal from the surface region.

\section{Terahertz characterization}

Further, we have investigated experimentally GaAs/AlAs structures as $\mathrm{THz}$ detectors by spectral photocurrent measurements. The data are given in Fig. 4. It is seen that with the increase of the radiation wavelength the photocurrent decreases. The mechanism responsible for the photocurrent signal is believed to be photothermal ionization of Be acceptors as all spectral curves correspond to the responsivity falling edge at photon energies below the acceptor binding energy. The observed responsivity edge extends to long wavelengths probably due to contribution of excited acceptor states $2 P_{3 / 2}$ and $2 P_{5 / 2}$ having transition energies several meV lower than the binding energy [4]. It should be noted that the absorption lines due to transitions to excited Be acceptor states were observed in [5]

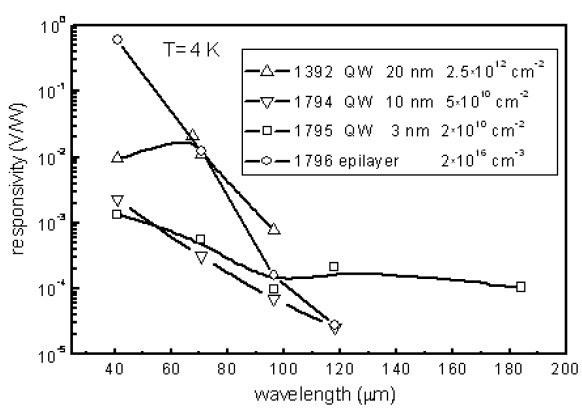

Fig. 4. Responsivity of the QW structures as a function of radiation wavelength at $4 \mathrm{~K}$. For comparison, data of Be-doped GaAs epilayer are depicted. 
on the same $\delta$-doped GaAs/AlAs QW structures as studied here. A spectral shift of responsivity to short wavelengths in sample 1794 is in good agreement with the influence of quantum confinement to the acceptor binding energy. Despite sample 1795 having the highest activation energy and similar doping, it shows extension of the photoresponse tail to longer wavelengths. We think that some additional photoconductivity may arise from photoexcitation of holes at crystal imperfections and centers capable to trap holes with low binding energies. In contrast, sample 1392 has about two orders of magnitude higher doping density, therefore, higher responsivity can be related to increased $\mathrm{THz}$ absorption. Furthermore, its high doping density may also induce a hopping effect observed in this spectral range between optically excited acceptor states [6] giving thus rise to an additional photosignal with smaller activation energies. As it is seen from Fig. 4, the experimental data supports the previous discussion. Finally, we show that PR, $\mathrm{SPV}$, and terahertz photocurrent methods can give valuable information about internal electric fields and electronic structure of $\delta$-doped multiple QW structures.

\section{Acknowledgments}

This work was supported, in part, by a NATO Collaborative Linkage Grant PST.CLG. 979121 "Semiconductor Nanostructures for Microwave to T-wave Technology", EU Center of Excellence in Processing, Research and Application of Advanced Materials (PRAMA) and the Lithuanian State Science and Studies Foundation under contract V-04004.

\section{References}

[1] W.M. Zheng, M.P. Halsall, P. Harmer, P. Harrison, M.J. Steer, Appl. Phys. Lett. 84, 735 (2004).

[2] Shouvik Datta, Sandip Ghosh, B.M. Arora, Rev. Sci. Instrum. 72, 177 (2001).

[3] L. Kronik, Y. Shapira, Surf. Sci. Rep. 39, 1 (1999).

[4] R.F. Kirkman, R.A. Stradling, P.J. Lin-Chung, J. Phys. C, Solid State Phys. 11, 419 (1978).

[5] W.M. Zheng, P. Halsall, P. Harrison, J.-P.R. Wells, I.V. Bradley, M.J. Steer, Appl. Phys. Lett. 83, 3719 (2003).

[6] C.J. Summers, R. Dingle, D.E. Hill, Phys. Rev. B 1, 1603 (1970). 